\title{
Mineral metabolism outcomes with frequent hemodialysis
}

More frequent and extended

hemodialysis is associated with a decrease in serum phosphorus levels and a reduced need for phosphorus binders according to an analysis of the Frequent Hemodialysis Network (FHN) Daily and Nocturnal Trials.

"The FHN Trials were done to determine whether or not dialyzing six times a week was better than dialyzing three times a week," says investigator John Daugirdas. Mineral metabolism was one of nine prespecified secondary outcomes in the original FHN Trials and Daugirdas et al. have now examined the effects of treatment assignment on predialysis serum phosphorus levels and on prescribed phosphorus binder dose.

Frequent daily hemodialysis (with hemodialysis sessions of 1.5-2.75 h duration six times weekly) was associated with a $0.15 \mathrm{mmol} / \mathrm{l}$ decrease in mean serum phosphorus levels at month 12 relative to conventional hemodialysis. Patients on frequent nocturnal hemodialysis (with hemodialysis sessions of $6-8 \mathrm{~h}$ duration six times weekly) had a $0.4 \mathrm{mmol} / \mathrm{l}$ relative decrease in mean serum phosphorus concentration. A higher baseline phosphorus concentration was associated with a more pronounced treatment effect in both trials.

\section{At month 12, frequent daily hemodialysis reduced the equivalent phosphorus binder dose ... by $0.96 \mathrm{~g}$ per day... 77}

At month 12, frequent daily hemodialysis reduced the equivalent phosphorus binder dose (expressed in terms of g calcium acetate) by $0.96 \mathrm{~g}$ per day from baseline versus an increase of $0.39 \mathrm{~g}$ per day in the conventional treatment arm. Almost three-quarters of participants assigned to frequent nocturnal hemodialysis achieved freedom from phosphorus binders compared with $8 \%$ of patients receiving conventional treatment. In fact, $60 \%$ of patients on frequent nocturnal hemodialysis who received $>35 \mathrm{~h}$ of dialysis per week required the addition of phosphorus to the dialyzate to prevent hypophosphatemia.

Treatment had no significant effect on serum calcium or parathyroid hormone (PTH) levels, although a trend for lower PTH levels in the frequent nocturnal hemodialysis arm and higher PTH levels in the frequent daily hemodialysis arm existed despite an increased dose of vitamin $\mathrm{D}$ derivative. The investigators found no evidence for an effect of treatment assignment on protein intake.

"More frequent hemodialysis and/or extended hemodialysis session length could be expected to improve phosphorus control in patients experiencing, or at risk for, important associated complications," conclude the authors.

Helene Myrvang

Original article Daugirdas, J. T. et al. Effects of frequent hemodialysis on measures of CKD mineral and bone disorder. J.Am. Soc. Nephrol. doi:10.1681/ ASN.2011070688 